ACCEPTED MANUSCRIPT

\title{
Toy geyser experiment: periodicity, operating conditions and coupling
}

To cite this article before publication: Martin Brandenbourger et al 2019 Eur. J. Phys. in press https://doi.org/10.1088/1361-6404/aafbf4

\section{Manuscript version: Accepted Manuscript}

Accepted Manuscript is "the version of the article accepted for publication including all changes made as a result of the peer review process, and which may also include the addition to the article by IOP Publishing of a header, an article ID, a cover sheet and/or an 'Accepted

Manuscript' watermark, but excluding any other editing, typesetting or other changes made by IOP Publishing and/or its licensors"

This Accepted Manuscript is @ 2018 European Physical Society.

During the embargo period (the 12 month period from the publication of the Version of Record of this article), the Accepted Manuscript is fully protected by copyright and cannot be reused or reposted elsewhere.

As the Version of Record of this article is going to be / has been published on a subscription basis, this Accepted Manuscript is available for reuse under a CC BY-NC-ND 3.0 licence after the 12 month embargo period.

After the embargo period, everyone is permitted to use copy and redistribute this article for non-commercial purposes only, provided that they adhere to all the terms of the licence https://creativecommons.org/licences/by-nc-nd/3.0

Although reasonable endeavours have been taken to obtain all necessary permissions from third parties to include their copyrighted content within this article, their full citation and copyright line may not be present in this Accepted Manuscript version. Before using any content from this article, please refer to the Version of Record on IOPscience once published for full citation and copyright details, as permissions will likely be required. All third party content is fully copyright protected, unless specifically stated otherwise in the figure caption in the Version of Record.

View the article online for updates and enhancements. 


\title{
Toy geyser experiment: periodicity, operating conditions and coupling
}

\author{
M Brandenbourger ${ }^{1,2}, \mathbf{S}$ Dorbolo ${ }^{1}$ and B Darbois Texier ${ }^{1,3}$ \\ ${ }^{1}$ GRASP, Physics Dept., University of Liège, B4000 Liège, Belgium. \\ ${ }^{2}$ Soft matter group, IOP, University of Amsterdam, Science Park 904 Amsterdam, 1098 XH \\ Netherlands. \\ ${ }^{3}$ SMAT-C, Departamento de Física de la Universidad de Santiago de Chile, Avenida Ecuador \\ 3493, 9170124 Estación Central, Santiago, Chile. \\ E-mail: s.dorbolo@uliege.be
}

\begin{abstract}
A natural geyser can be reproduced by a toy experiment composed of a water pool located above a water reservoir, the two being connected by a long and narrow tube. When the bottom reservoir is heated, the system may experience periodic eruptions of hot water and steam at the top similarly to the geyser effect occurring in nature. The eruption frequency of a toy geyser is inspected experimentally as a function of the heating power and the height of the setup. We propose a thermodynamic model of this system that predicts the time between eruptions. A phase diagram that takes into account the thermal energy provided to the geyser and the geometry was constructed for the toy geyser. The conditions to obtain the geyser effect have been determined. The study of the toy geyser is then extended to the case of two reservoirs connected to the same tube. Such a coupled system adopts a complex time-evolution that reflects the dynamics of natural geysers. We analyze the behavior of a toy geyser with two reservoirs by the way of statistical tools and develop a theoretical model in order to rationalize our observations.
\end{abstract}

\section{Introduction}

A geyser can be defined as a periodically eruptive spring resulting from Earth's thermal activity. One of the most famous geyser is Old Faithful [Fig. 1(a)] in Yellowstone National Park in the United States, which has raised the fascination of millions of visitors over the years. It erupts about every 90 minutes and projects hot water up to $56 \mathrm{~m}$ high $[1,2]$. Probably the first scientist to describe the principle of a geyser was Robert Bunsen in 1845 after the Danish government sponsored him to study the eruption of Mount Hekla in Iceland [3]. He lowered a thermometer into geyser depths and discovered a reservoir of "superheated" water at its base. He understood that when the water in the reservoir boils, the liquid in the conduit is ejected upwards and produces an eruption of hot water and steam. Bunsen and his student John Tyndall built a toy geyser based on the same principle as illustrated in Fig. 1(b) [4]. Such a demonstrative experiment showed eruptions regularly spaced in time and confirmed their intuitions on the fundamental principles underlying natural geysers. The realization of a geyser at reduced scale promotes Bunsen's theory for geyser among the geophysical community [5]. Nowadays, similar setups are present in various Science Museum like the Exploratorium in San Frańcisco (USA) and Source-o-Rama in Chaudfontaine (BE) and can be easily reproduced 
at home with a pressure cooker and a hot plate (Caution: to make this experiment safe, all the connexions of the piping system have to be sealed and one has to stay away $(\gtrsim 20 \mathrm{~cm})$ of the hot reservoir and the erupting vent) [6]. We are convinced that the experiment can be shown from the primary school to undergraduate students with different level of comprehension. The basic measurement procedure only requires a stop watch. Moreover, this setup can be implemented by undergraduate students in the context of a guided experimental project as two thermocouples are sufficient to characterize the dynamic of the system and the risks associated to the manipulation are very limited. Thus, the toy geyser setup is the perfect experiment to become more familiar with thermal transfers, boiling processes and fluid dynamics at an affordable cost. Moreover, the connection between this system and a spectacular geothermal phenomenon may raise the attention of students and curious people towards science. Lasic reproduced a toy geyser experiment and studied the time evolution of the temperature and the pressure inside the reservoir [7]. This work identifies the different phases of a toy geyser eruption and discusses the associated temperature and pressure signals. Steinberg et al. conducted similar experiments and provided a theoretical model in order to describe the geysering dynamics $[8,9]$. Lately, Toramaru and Maeda focused on the eruption style and the erupted mass depending on the system geometry [10]. The influence of a bubble trap in the vertical conduit of a toy geyser was investigated by Adelstein et al. [11]. More recently, the pressure and temperature variations in a toy geyser system were carefully studied by Flieller/et al. [6]. However, despite the deep understanding of toy geyser systems provided by these studies, the exact conditions allowing the succession of eruptions and reservoir's refills remains unexplored.

Furthermore, the natural geysers erupt with much more complex time series than the one displayed by a toy geyser, as revealed by in situ data collection in Yellowstone USA [1, 2, 12], El Tatio in Chile $[13,14]$ or Atami in Japan [15]. Such a complexity can be attributed to a high sensitivity of geysers dynamics towards water and steam inflow [14], barometric pressure, tectonic stresses $[16,17,18]$, reservoirs interconnections [19] or the presence of dissolved gases $[20,21]$. Over the past decades, crucial steps in the theoretical modelling of geyser eruptions have been achieved [22, 23, 24]. However, these studies were mainly focused on specific geysers rather than laboratory experiments, which did not allow to easily quantify the influence of the physical parameters.

Throughout this paper we aim to describe how a toy geyser experiment can be implemented at a low cost and how scientific methods and notions can be approached with this setup. We study and predict the dynamics of a toy geyser, to understand conditions under which it operates and to reveal the similarities between this setup and natural geysers. The erupting dynamics of this system has been inspected as a function of the injected heat power and its geometrical characteristics. The different stages of a geyser eruption and the evolution of the reservoir temperature with time are described in Section 2. In Section 3 we develop a theoretical model based on thermodynamics and fluid dynamics in order to rationalize our observations. The conditions required to produce a geyser effect are inspected in Section 4. Indeed, we observed that the geyser effect can yanish because of intense vaporization or thermal convection in the conduit. Finally, in Section 5 we consider a double toy geyser with two interconnected reservoirs in order to approach the degree of complexity of natural systems.

\section{Experiments}

\subsection{Experimental set-up and driving parameters}

The toy geyser was constructed with a glass reservoir $(1.2 \mathrm{~L})$ connected to a pool $(4.0 \mathrm{~L})$ through a long and harrow vertical conduit (silicone tube) as sketched in Fig. 1(c). The system was filled with water. We denote $H$ the total height of the liquid column from the bottom of the reservoir to the liquid surface. The depth of the pool is $h$, the height of the reservoir $h^{\prime}$ and the internal conduit radius $R$. 
(a)

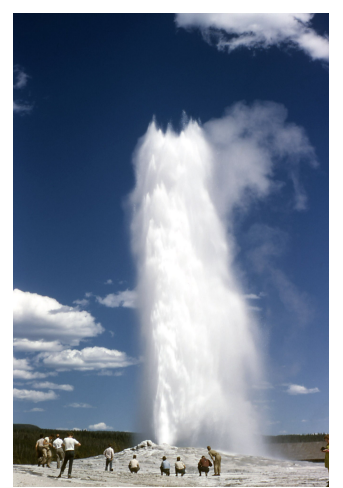

(b)

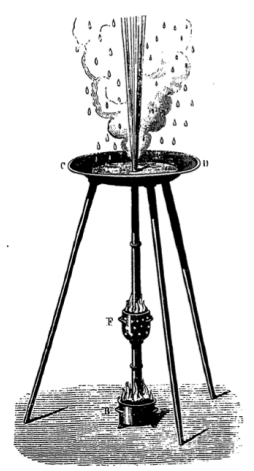

(c)

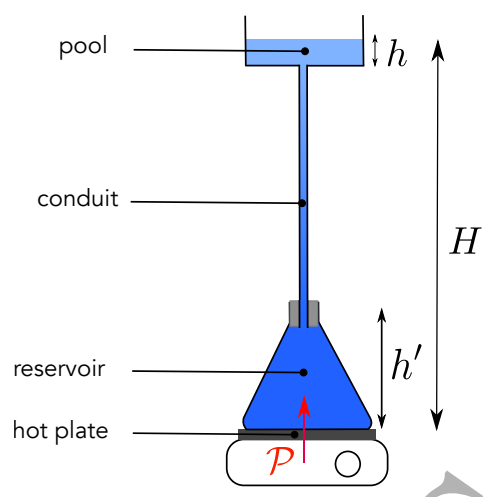

(d)

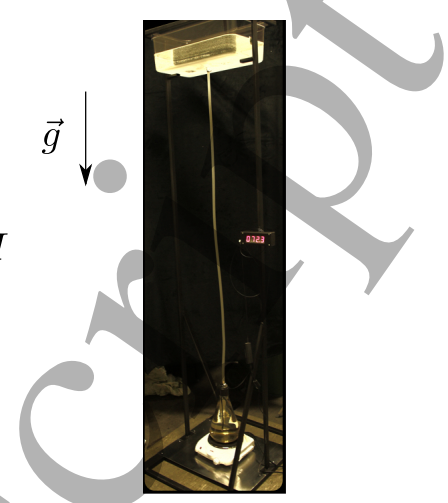

Figure 1. (a) People watching Old Faithful erupt from a geyser cone, Yellowstone National Park, 1948. (b) Toy model of a geyser by Tyndall [25]. (c) Sketch of the experimental setup. The glass reservoir containing a mass $M$ of water at a temperature $T_{r}$ was connected to the pool by a long conduit of radius $R$. The water in the pool was maintained at a constant temperature $T_{p}$. The system was completely filled with a column of water of height $H$ chosen between 1.6 and $11.5 \mathrm{~m}$, the pool had a depth $h$ and the reservoir had a height $h^{\prime}$. The heating plate provided a specific heat power $\mathcal{P}$ to the water of the reservoir. (d) Photo of the experimental setup.

The reservoir was heated from below by a hot plate delivering a constant heat power. As the hot plate is not insulated from the rest, the effective heat power was experimentally determined as follows. The reservoir was filled with water. A thermistor plunged inside the water allowed measuring the increase of temperature of the water with respect to time. The power delivered by the hot plate must be sufficient to observe a linear increase of the temperature with time, i.e. $T_{r}=\chi t$. The mass of water $M$ being known, the heat power $\mathcal{P}$ is given by $\chi M c$ where $c=4186 \mathrm{~J} \mathrm{~K}^{-1} \mathrm{~kg}^{-1}$ is the specific heat of the water. In our experiments, $\mathcal{P}$ was tuned between 30 and $220 \mathrm{~W} / \mathrm{kg}$. Before starting the geyser experiments, one can already infer that if the power delivered by the plate is not sufficient, the boiling and geyser effect will not be observed. Thus, the specific heat power is the natural driving parameter for the observation of a geyser.

As liquid flow plays a major role during an eruption, the dimensions of the tube connecting the reservoir and the pool is the second driving parameter of the system. We varied the length and the diameter of the tube according to Tab. 1. Our basis configuration (Exp.1) is a geyser with a total height $H$ of $1.6 \mathrm{~m}$ and a tube of $3 \mathrm{~mm}$ of inner radius.

\begin{tabular}{|c|c|c|c|c|c|c|c|}
\hline Exp. & $H(\mathrm{~m})$ & $R(\mathrm{~mm})$ & $M(\mathrm{~kg})$ & $h(\mathrm{~cm})$ & $h^{\prime}(\mathrm{cm})$ & $\mathcal{P}(\mathrm{W} / \mathrm{kg})$ & Geysering \\
\hline 1 & 1.6 & 3.0 & 1.2 & 4.0 & 25 & $30 \rightarrow 220$ & yes \\
2 & 11.5 & 3.0 & 1.2 & 4.0 & 25 & $30 \rightarrow 220$ & yes \\
3 & 1.6 & 25.0 & 1.2 & 4.0 & 25 & $30 \rightarrow 220$ & no \\
\hline
\end{tabular}

Table 1. Sets of experimental parameters.

To start measurement procedures, the system must be completely filled with water. Any bubble cannot be tolerated in the conduit or in the reservoir. This may be tricky regarding the small diameter of the conduit in Exp.1. Here is the preliminary procedure to completely fill the system. Note that the procedure is similar to the geyser effect. First, the reservoir is filled with water. Then, the conduit is connected to the top of the reservoir and to the bottom of the pool. Water is then poured in the pool $(h \simeq 4 \mathrm{~cm})$. The liquid does not enter the conduit 
because of the air pressure in the tube. The system has to be initiated by heating the reservoir until boiling. When boiling, the air in the conduit is ejected and replaced by steam. For safety reasons, the experiment should always be designed with a large enough pool $(20 \times 20 \mathrm{~cm})$, such as the ejection of hot water and steam is confined to the experiment. The heating source is then to be switched off. The boiling stops and the water from the pool is sucked down in the reservoir and the conduit. After the cooling of the geyser to room temperature, the whole system is ready for operation.

\subsection{Geyser observation}

We first injected enough specific heat to observe the boiling of the water in the reservoir. A First experiment was conducted for a specific heating power $\mathcal{P}=130 \mathrm{~W} / \mathrm{kg}$. Under these conditions, the toy geyser undergoes periodic erupting events spaced between quiet phases. The time between two successive eruptions was $5 \mathrm{~min}$ and $27 \mathrm{~s} \pm 4 \mathrm{~s}$. This periodic behavior is illustrated by the evolution of the temperature $T_{r}$ measured every second in the middle of the reservoir as shown in Fig. 2(a). After a first heating phase, the temperature in the reservoir follows a saw-tooth evolution between about $93^{\circ} \mathrm{C}$ and $102^{\circ} \mathrm{C}$. The red bullets represents the moment at which the geyser ejects steam and water.

(a)
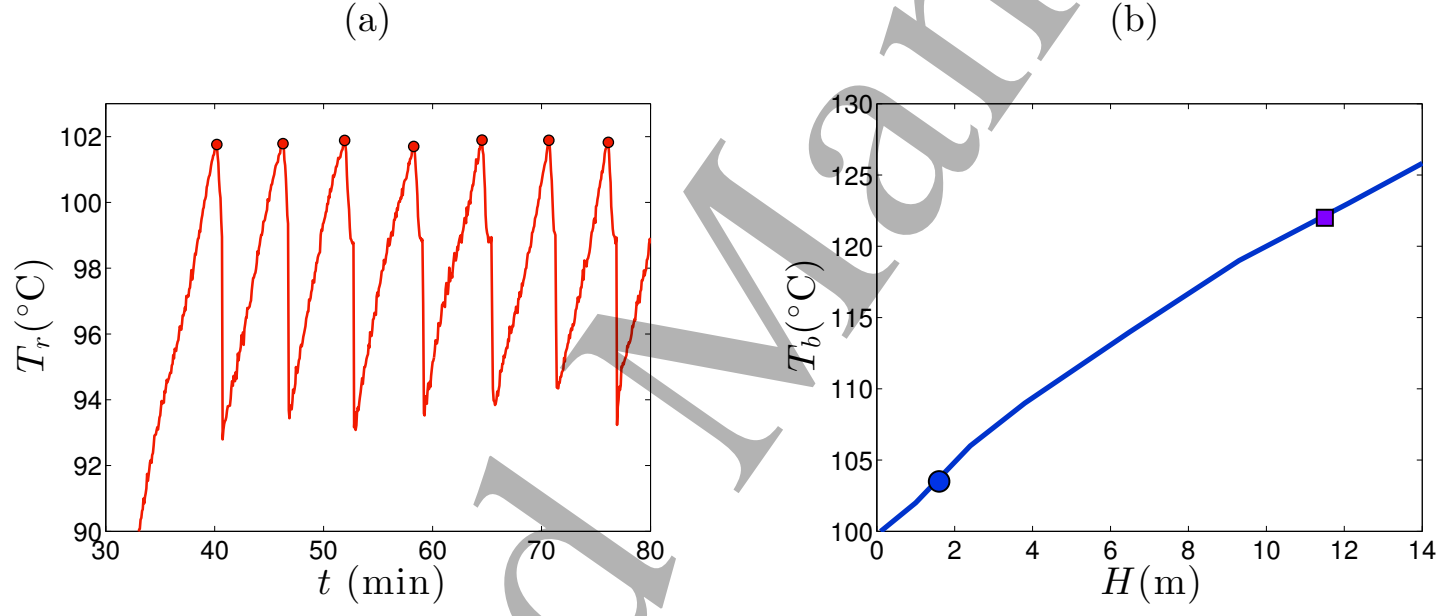

Figure 2. (a) Evolution of the temperature $T_{r}$ in the reservoir as a function of time for a toy geyser of height $H=1.60 \mathrm{~m}$, specific heating power $\mathcal{P}=130 \mathrm{~W} / \mathrm{kg}$, pool height $h=4.0$ $\mathrm{cm}$, reservoir height $h^{\prime} \Rightarrow 25 \mathrm{~cm}$ and conduit radius $R=3.0 \mathrm{~mm}$. Dots indicate the time at which eruptions begin. (b) Boiling temperature $T_{b}$ of water as a function of the height $H$ of the overlying column extracted from [26]. Symbols show the the two conduit heights used in this study.

At this point, we can define three characteristic periods of time: the time $\tau$ between two eruption starts, the duration of the eruption $\tau_{e}$ and the heating time $\tau_{h}$ corresponding to the time spent between the end of an eruption and the start of the following one. These durations are linked to each other according to $\tau=\tau_{e}+\tau_{h}$. The variation of the reservoir temperature during one period, $T_{r}(t)$, is reported schematically in Fig. 3 and consists in three steps: the heating, the eruption, the return to the initial state.

Heating- The water in the reservoir is heated up from $T_{r}\left(t_{1}\right)$ at $t=t_{1}$ to the boiling point $T_{b}(H)$ reached at $t=t_{2}$ [stage (a) in Fig. 3]. The boiling temperature $T_{b}(H)$ depends on the pressure applied by the water column (i.e. $\rho g H$ where $\rho$ is the water density and $g$ the gravitation acceleration). The boiling temperature of water as a function of the height of the oyerlying column $H$ is shown in Fig. 2(b) and is equal to $102^{\circ} \mathrm{C}$ for $H=1.60 \mathrm{~m}$. When the 


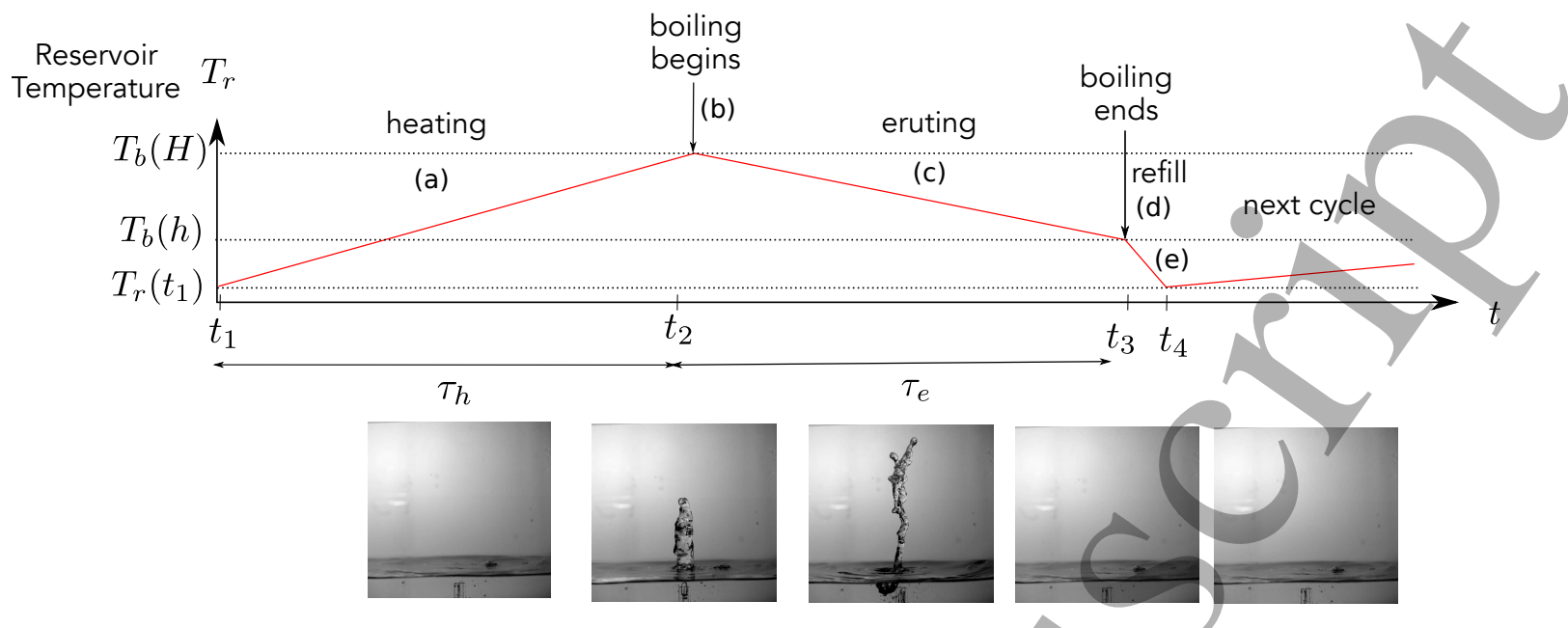

(a)

(b)

(c)

(d)

(e)

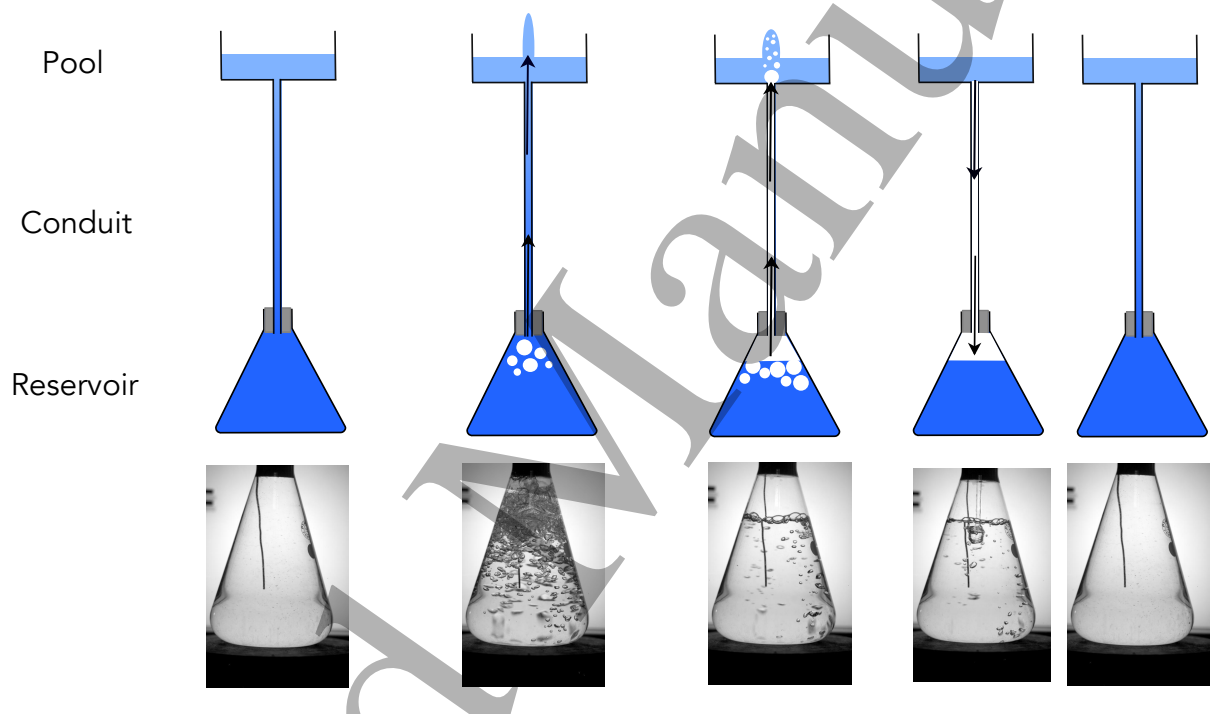

Figure 3. Sketch showing the different stages of the cycle of a toy geyser: (a) Heating the reservoir from $T_{r}\left(t_{1}\right)$ at $t=t_{1}$ up to the boiling temperature $T_{b}(H)$. (b) Water boiling in the reservoir provokes an eruption at $t=t_{2}$. (c) Vapor emission throughout the conduit. (d) End of the eruption at $t=t_{3}$, the water from the pool refills the reservoir. (e) The system had returned to its initial state at $t=t_{4}$. The typical evolution of the reservoir temperature $T_{r}$ over time is described by the red solid line in the top graph. The pool temperature remains constant at $T_{p}=30^{\circ} \mathrm{C}$. Bottom and top pictures correspond respectively to the reservoir and to the pool at different moments of the geyser cycle.

water in the reservoir reaches the corresponding boiling point (at $t=t_{2}$ ), an intense production of vapor bubbles occurs. The heating step lasts $\tau_{h}=t_{2}-t_{1}$ and ends when the geyser starts to erupt.

Eruption- Buoyancy induces the upward motion of vapor bubbles in the conduit. These vapor bubbles entrain the water and produce an eruption of hot water and steam at the top of the toy geyser [stage (b) in Fig. 3]. Ejected water can reach a height of $20 \mathrm{~cm}$ above the pool surface for the set of experimental parameters we used ( $c f$. Table 1). After the ejection of the liquid from the conduit, the water in the reservoir keeps on boiling and the vapor continues to eseape through the conduit toward the pool, making loud noise [stage (c) in Fig. 3]. The 
total amount of water transferred from the reservoir to the pool during the erupting duration (excluding the water contained in the conduit) is denoted $\Delta M$. As the boiling goes on, the vapor production cools down the water remaining in the reservoir. When this latter reaches the boiling temperature $T_{b}(h)$, corresponding to the pressure of a water column of height $h$, the vapor emission decreases till the vapor flow cannot resist the entry of water from the pool into the conduit [stage (d) in Fig. 3 at $t=t_{3}$ ]. At that point, the water falls back into the reservoir and the eruption phase is finished. The eruptions lasted a time $\tau_{e}=t_{3}-t_{2}$.

Return to initial state- After the eruption at $t=t_{4}$, the temperature in the reservoir reaches again $T_{r}\left(t_{1}\right)$, the equilibrium temperature resulting from the mixture of a mass of water $M-\Delta M$ at the temperature $T_{b}(h)$ and a mass of water $\Delta M$ coming from the pool at a temperature $T_{p}$ [stage (e) in Fig. 3]. This stage is also corroborated by recent measurements of the pressure in the reservoir [27]. After that, the temperature in the reservoir increases from $T_{r}\left(t_{1}\right)$ to $T_{b}(H)$ in a time $\tau_{h}$ before an other eruption starts. It is important to underline that it takes several cycles to obtain the same initial state. Indeed, due to the contact with the ambient air and liquid replacement in between each eruption, the observed temperature of the pool slowly increased from room temperature to a constant temperature of $T_{p}=30 \pm 2^{\circ} \mathrm{C}$. All the measurements presented thereafter are realized in the regime of constant pool temperature.

\subsection{Measurements}

During the eruption of a toy geyser, the two characteristic eruption times $\tau_{h}, \tau_{e}$ and the exchanged mass $\Delta M$ were easily measured with a chronometer and a graduated reservoir. In this study, they were measured as a function of the specific heating power $\mathcal{P}$ and the geyser heights $H(H=1.6 \mathrm{~m}$ and $H=11.50 \mathrm{~m})$. The boiling temperature of water associated with each height is respectively $102^{\circ} \mathrm{C}$ and $120^{\circ} \mathrm{C}$ for a standard outside atmospheric pressure [Fig. 2(b)]. The mean values of $\tau_{h}, \tau_{e}$ and $\Delta M$ were determined over more than 10 events and the experimental results are shown in Fig. 4.

The figures $4(\mathrm{a})$ and $(\mathrm{b})$ present the heating duration $\tau_{h}$ and the erupting duration $\tau_{e}$ as a function of the specific heat power respectively. The measurements are reported for the two considered geometries, i.e. $H=1.6 \mathrm{~m}$ and $11.5 \mathrm{~m}$ represented by blue dots and purple squares respectively. From Fig.4(a), one deduces that the values of $\tau_{h}$ ranges from 2 to $30 \mathrm{~min}$. This characteristic time increases with the geyser height $H$ and decreases with the specific heating power $\mathcal{P}$. The logarithmic scale diagram in Fig. 4(a) reveals that the time $\tau_{h}$ is inversely proportional to $\mathcal{P}$. In Fig. 4(b), the erupting time $\tau_{e}$ is presented as a function of the specific heat power for the two considered geometries. This characteristic time increases with the conduit height $H$ and shows a small dependency with the specific heating power $\mathcal{P}$. The average erupting times are $50 \mathrm{~s}$ and $90 \mathrm{~s}$ for $H=1.6 \mathrm{~m}$ and $11.5 \mathrm{~m}$ respectively.

Figure $4(\mathrm{c})$ shows the exchanged mass fraction $\Delta M / M$ as a function of the specific heating power and the water column height. The exchanged mass $\Delta M$ was measured by recording the minimal water level reached in the reservoir during an eruption with a high speed camera. One notices in Fig. 4(c) that $\Delta M / M$ increases both with $\mathcal{P}$ and $H$.

\section{Model}

From the observations, we model the heating time $\tau_{h}$ (section 3.1), the erupting duration $\tau_{e}$ (section 3.2), and the variation of water volume $\Delta M$ in the reservoir (section 3.3).

\subsection{Sleeping time: $\tau_{h}$}

The time between the end of an eruption and the start of the next one corresponds to the time needed to heat the water in the reservoir from the initial temperature $T_{r}\left(t_{1}\right)$ to the boiling temperature $T_{b}(H)$ (corresponding to a water column of height $H$ [Fig. 2(b)]). If a specific heat power $\mathcal{P}$ is transferred to the reservoir, its temperature increases from $T_{r}\left(t_{1}\right)$ to $T_{b}(H)$ in a time 
(a)

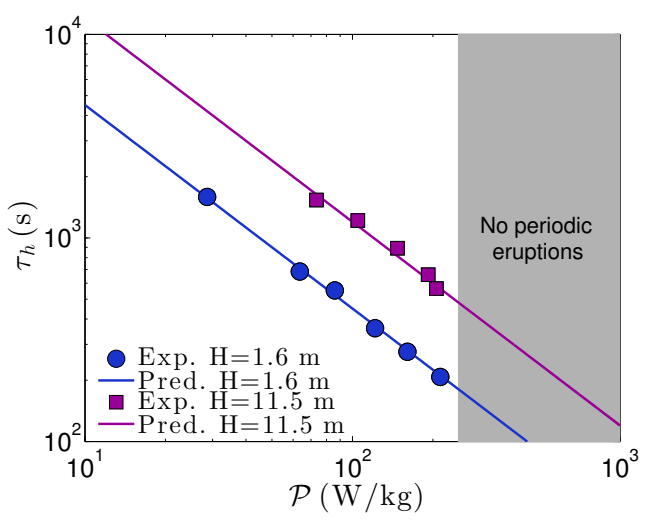

(b)

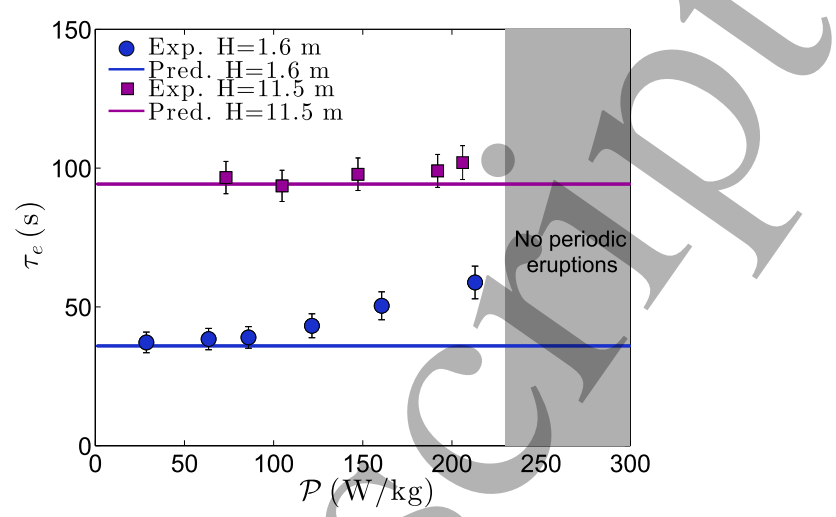

(c)

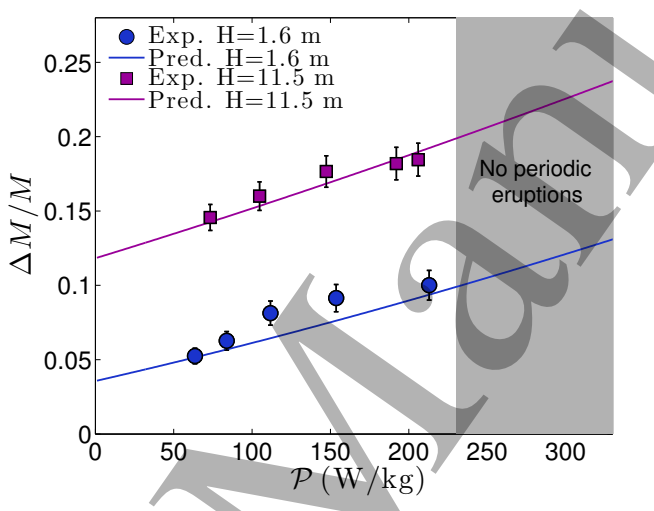

Figure 4. (a) Mean values of the heating time $\tau_{h}$ as a function of the specific heat power $\mathcal{P}$ for two different water column heights $H=1.6 \mathrm{~m}$ and $H=11.5 \mathrm{~m}$ (logarithmic scales). Symbols correspond to experiments and solid lines show the predictions of Eq. (3) detailed in Section 3. (b) Mean erupting time $\tau_{e}$ as a function of the specific heat power $\mathcal{P}$ for $H=1.6 \mathrm{~m}$ and $H=11.5 \mathrm{~m}$. Symbols correspond to experiments and solid lines show the predictions of Eq. (4) deduced from the model developed in Section 3. (c) Mean mass fraction $\Delta M / M$ exchanged between the reservoir and the pool during an eruption as a function of the specific heating power $\mathcal{P}$ for $H=1.6 \mathrm{~m}$ and $H=11.5 \mathrm{~m}$. Symbols correspond to experiments and solid lines show the predictions of Eq. (7) issued from the model explained in Section 3. In the three plots, the grey zones indicate where no periodid eruptions have been observed.

$$
\tau_{h}=\frac{c}{\mathcal{P}}\left[T_{b}(H)-T_{r}\left(t_{1}\right)\right] .
$$

During an eruption, a mass of water $\Delta M$ moves from the reservoir to the pool [Fig. 5]. The eruption stops when the temperature of the water remaining in the reservoir is close to the boiling temperature $T_{b}(h)$ corresponding to the pressure imposed by a water column of height $h$. When the vapor production is not sufficient any longer to counterbalance the pressure imposed by the height $h$ of liquid in the pool, the liquid flows from the pool to the reservoir. Thus, a mass $\Delta M$ at $T=T_{p}$ moves from the pool to the reservoir and fills it completely. The temperature $T_{r}\left(t_{1}\right)$ of the reservoir at this particular time is 


$$
T_{r}\left(t_{1}\right)=\frac{(M-\Delta M) T_{b}(h)+\Delta M T_{p}}{M}
$$

Combining Eqs. (1) and (2) yields

$$
\tau_{h}=\frac{c}{\mathcal{P}}\left[\frac{\Delta M}{M}\left(T_{b}(h)-T_{p}\right)+\left(T_{b}(H)-T_{b}(h)\right)\right]
$$

Equation (3) is divided in two terms. The first part of the equation corresponds to the cooling of the reservoir by the entry of a mass $\Delta M$ of water coming from the pool maintained at a temperature $T_{p}$. The second part of the equation is the time required to reach the boiling temperature $T_{b}(H)$ below a water column of height $H$. These contributions imply that the geyser height $H$, the pool temperature $T_{p}$ and the geometry of the plumbing system impact the time between eruptions. In our experiments, all quantities involved in Eq. (3) are known except $\Delta M / M$, which has been measured and interpolated (polynomial interpolation). Therefore, an estimation of the time between eruptions $\tau_{h}$ can be deduced. Such predictions are plotted as solid lines in Fig. 4(a) for the two geyser heights studied. The agreement between experiments and predictions validates the approach considered above.

\subsection{Eruption time, $\tau_{e}$}

After the beginning of an eruption, once the water is ejected from the conduit, the temperature of the reservoir is $T_{b}(H)$ whereas the overlying pressure is close to the atmospheric pressure, which imposes an equilibrium temperature $T_{b}(h)$. The erupting time is associated with the time needed to cool the reservoir from $T_{b}(H)$ to $T_{b}(h)$ by vaporizing water [stage (c) in Fig. 3]. In our situation, we assume that this process is limited by the thermal transfer occurring in the reservoir with bubbles forming on nucleation sites, their growth and their ascension. Such a thermal transfer has been described in detail by Pinhasi et al.[28] and by Lu and Kieffer in particular cases of geothermal interest [21]. In the following, we approach the complexity of boiling processes through an heuristic equation that expresses the power lost by vaporization as a power law of the temperature difference, i.e. $\dot{Q}=\beta\left(T_{r}-T_{b}(h)\right)^{\gamma}$ [Fig. 5]. In a first approximation, the power lost by vaporization is balanced by the temperature variation of the reservoir $M c \dot{T}_{r}=-\beta\left(T_{r}-T_{b}(h)\right)^{\gamma}$. Solving the previous equation from a reservoir temperature $T_{b}(H)$ to a threshold temperature $T_{t}$ at which an eruption stops provides the following prediction for the erupting duration

$$
\tau_{e}=\frac{M_{c}}{\beta(1-\gamma)}\left[\left(T_{b}(H)-T_{b}(h)\right)^{1-\gamma}-\left(T_{t}-T_{b}(h)\right)^{1-\gamma}\right] .
$$

We note that the erupting duration predicted by Eq. (4) increases with the geyser height as observed experimentally in Fig. 4(b). More quantitatively, this model was adjusted to the experimental data, Solid lines in Fig. 4(b) correspond to the best fit of experiment data with the predictions of Eq. (4) for $T_{t}-T_{b}(h)=0.1^{\circ} \mathrm{C}, \gamma=0.64$ and $\beta=3.7 \times 10^{2} \mathrm{~W} \mathrm{~K}^{-0.64}$. The deviation of experimental data from the theoretical predictions at large heating power $(\mathcal{P}>150$ $\mathrm{W} / \mathrm{kg}$ ) is attributed to the omission of the energy injected by the heating plate during $\tau_{e}$ in the energy balance of the model. Indeed, the specific heating power $\mathcal{P}$ delays the cooling of the water in the reservoir.

\subsection{Exchanged mass, $\Delta M$}

When an eruption has occurred and the water has left the conduit, the vaporization in the reservoir results from both the injected heat power $\mathcal{P} M$ and the heat flux $\dot{Q}$ coming from the water superheat [Fig. 5]. Thus, the power balance in this case writes 
heating

(a)

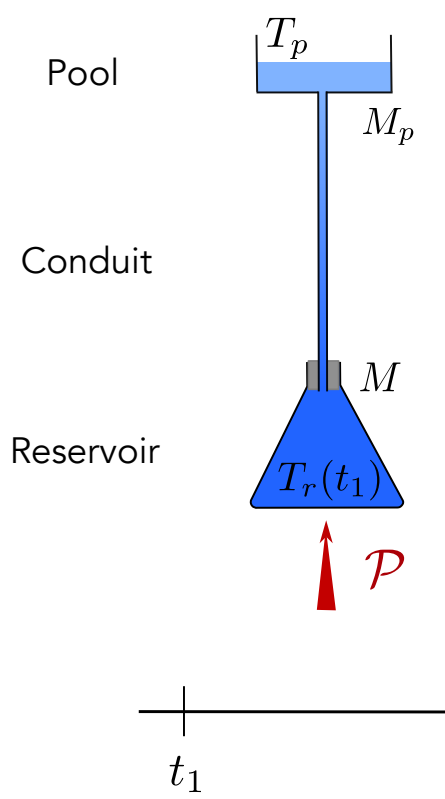

(b)

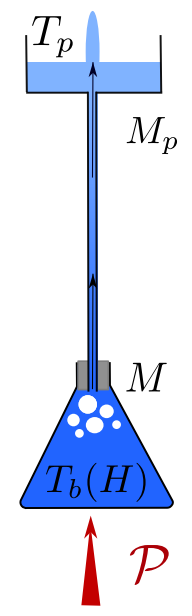

(c) refill

(d)

(e)

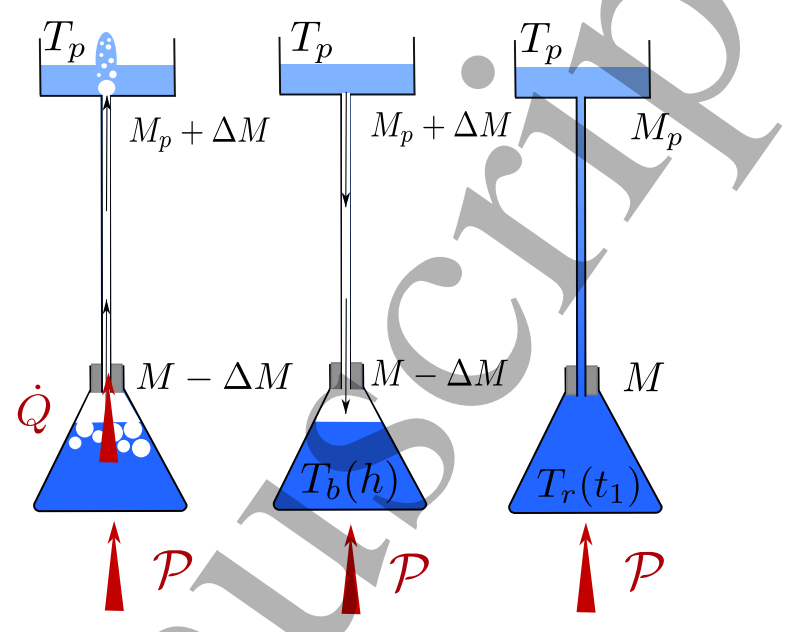

Figure 5. Sketch of the mass and heat transfers during a cycle of a toy geyser. At $t=t_{2}$ a mass $\Delta M$ of water is transferred from the reservoir to the pool and returns to the reservoir at $t=t_{3}$. A heat power $\mathcal{P}$ is constantly provided by the heat plate to the reservoir. Between $t=t_{2}$ and $t=t_{3}$, the boiling processes inside the reservoir of superheated water induce a leaving heat flux $\dot{Q}$.

$$
\mathcal{L} \dot{M}=\mathcal{P} M+\beta\left(T_{b}(H)-T_{b}(h)\right)^{\gamma}
$$

where $\dot{M}$ is the mass flow rate of vapor produced in the reservoir and $\mathcal{L}$ the latent heat of vaporization of water. The mass flow rate can be modeled by describing the vapor bubbles that grow and rise in the reservoir. Due to the constriction of the reservoir, the bubble volume fraction $\phi(z)$ increases along the vertical axis $z$ as sketched in Fig. 6. When the volume fraction reaches a critical value $\phi_{c}$, the bubbles coalesce and finally form the vapor phase. Considering that $\phi_{c}$ corresponds to the fraction at which a single bubble cannot be added to a cubic closepacked arrangement of monodispered bubbles, we estimate that $\phi_{c} \simeq 0.33$ consistently with the approach of Kaichiro and Ishii [29]. Thereby, the equilibrium position $z_{e}$ of the liquid/vapor interface during an eruption can be deduced from the critical bubble volume fraction in the liquid. Introducing the bubbles rising velocity $U_{b}$ in the reservoir, the vapor density $\rho_{v}$ and $R_{r}(z)$ is the radius of the reservoir at the altitude $z$ relatively to the bottom of the reservoir, we get

$$
\mathcal{L} \rho_{v} \phi(z) \pi R_{r}^{2}(z) U_{b}=\mathcal{P} M+\beta\left(T_{b}(H)-T_{b}(h)\right)^{\gamma} .
$$

Haberman and Morton showed that the terminal velocity of vapor bubbles in water is between $20 \mathrm{~cm} / \mathrm{s}$ and $30 \mathrm{~cm} / \mathrm{s}$ for bubble of radius comprised between $0.5 \mathrm{~mm}$ and $15 \mathrm{~mm}$ [30]. For the sake of simplicity, we consider that all the bubbles in the conduit rise at the same velocity $U_{b} \simeq 25 \mathrm{~cm} / \mathrm{s}$, which is consistent with our observations. The critical bubble volume fraction $\phi_{c}$ is reached for a reservoir radius 


$$
R_{r}\left(z_{e}\right)=\sqrt{\frac{\mathcal{P} M+\beta\left(T_{b}(H)-T_{b}(h)\right)^{\gamma}}{\pi \mathcal{L} \rho_{v} \phi_{c} U_{b}}} .
$$

This radius represents the constriction size at which the continuous vapor phase appears in the reservoir during the eruption. We deduce from Eq. (7) and geometrical considerations the mass of water $\Delta M$ ejected out of the reservoir during an eruption for the parameters corresponding/ to the experiments of section $2\left(M=1.2 \mathrm{~kg}, \rho_{v}=0.9 \mathrm{~kg} / \mathrm{m}^{3}, \mathcal{L}=2.6 \times 10^{6} \mathrm{~J} / \mathrm{kg}, \gamma=0.64\right.$ and $\beta=3.7 \times 10^{2} \mathrm{~W} \mathrm{~K}^{-0.64}$ ) and a critical bubble volume fraction $\phi_{c}=0.33$. The predictions of this approach are shown in Fig. 4(c) as solid lines. A relative agreement between the theory and experimental data is observed despite the strong assumptions we made concerning the constant velocity of ascending bubble and the rough estimation of the critical gas volumic fraction.

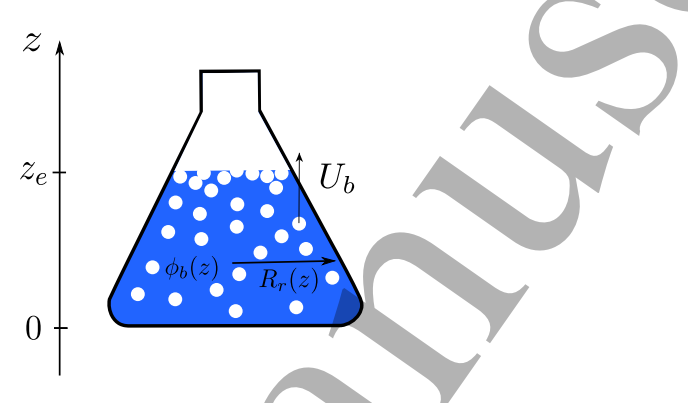

Figure 6. Definition of the parameters used in the model developed in section 3.3. All the vapor bubbles have the same upwards velocity $U_{b}$. Due to the reduction of the reservoir radius $R_{r}(z)$ with increasing height $z$, the bubble volume fraction $\phi(z)$ increases along this direction up to a critical volume fraction $\phi_{c}$ which defines the equilibrium position $z_{e}$ of the liquid/gas interface.

\section{Conditions for geysers existence}

The aim of this section is to inspect the conditions under which a toy geyser follows periodic eruptions. We identify the physical mechanisms associated to geyser eruptions and deduce a prediction for the frontiers of the different regimes undergone by the system : the geyser, the fumarole, the hot spring and the boiling pool regimes. Finally, these regimes are summarized in a phase diagram.

\subsection{Geyser's birth: bubble clogging}

First, we investigate the effect of the conduit radius on the toy geyser operations. Experimentally, we observe that if the conduit radius is too large $(R \gtrsim 25 \mathrm{~mm})$, the vapor bubbles produced by the boiling in the reseryoir are able to rise without provoking a clog in the conduit and no eruptions occur ( $c f$. Table 1). Thus, the initiation of a geyser eruption is caused by the transition from a dispersed flow regime to an annular flow regime. The different flow regimes of a liquid/gas mixture in a vertical conduit have been extensively studied by Mishima and Hibiki [31]. Furthermore, Kaichiro and Ishii showed that the transitions between different flow regimes are virtually not impacted by replacing air with steam [29]. The transition between the dispersed to the annular flow in the conduit can be approached with a critical gas volume fraction above which bubbles clog and entrain the water above. In the present situation, the vapor volume fraction in the conduit $\phi$ can be estimated by assuming that when the reservoir reaches its boiling temperature $T_{b}(H)$, all the specific heating power $\mathcal{P}$ is used to vaporize water. The balance between the vapor departure through the conduit and the vapor production in the reservoir yields 


\subsection{Geyser's death: transition to fumarole}

When varying the water level in the pool, $h$, we observed that, for small heights, a first eruption could be followed by a continuous vaporization of the water in the reservoir, without any further eruptions. In this case, the liquid in the pool cannot go down into the reservoir. Because of permanent emission of vapor through the conduit, this situation is called the "fumarole" regime. We noticed that a toy geyser operating in a fumarole regime can be brought back to a periodic regime by increasing the height $h$ of the water in the pool. Thereafter, we discuss quantitatively the minimal pool height that leads to a periodic regime. During an eruption, when the water in the reservoir has reached its equilibrium temperature $T_{b}(h)$, the specific heating power $\mathcal{P}$ induces water vaporization. Thus, the mass of water vapor produced per unit time is $\dot{M}=\mathcal{P} M / \mathcal{L}$ and it results a mean gas velocity in the conduit $U_{v}=\mathcal{P} M / \pi R^{2} \mathcal{L} \rho_{v}$. For the range of parameters used in our experiments, the order of magnitude of the gas velocity is $\sim 1 \mathrm{~m} / \mathrm{s}$ and the Reynolds number associated to the gas flow in the conduit is about $\sim 10^{3}$. As a consequence, we consider the dynamic pressure $\rho_{v} U_{v}^{2} / 2$ associated to the vapor flow at the exit of the conduit and we compare it with the hydrostatic pressure $\rho_{l} g h$ (where $\rho_{l}$ is the water density) at the bottom of the pool. If $\rho_{v} U_{v}^{2} / 2>\rho_{l} g h$, the water will not re-entry in the conduit and the system will stay in a fumarole regime. The critical specific heating power $\mathcal{P}_{c}$ above which the system does not follows periodic eruptions is

$$
\mathcal{P}_{c}=\pi \mathcal{L} R^{2} \sqrt{2 \rho_{v} \rho_{l} g h} / M
$$

For $R=3.0 \mathrm{~mm}, h=40 \mathrm{~mm}, \mathcal{L}=2.3 \times 10^{6} \mathrm{~J} / \mathrm{kg}, \rho_{v}=0.9 \mathrm{~kg} / \mathrm{m}^{3}, \rho_{l}=960 \mathrm{~kg} / \mathrm{m}^{3}$ and $M=1.2 \mathrm{~kg}$, this relation gives a critical value of $\mathcal{P}_{c} \simeq 1400 \mathrm{~W} / \mathrm{kg}$. In the experiments presented in section 2, the maximal specific heat power is $\mathcal{P}=220 \mathrm{~W} / \mathrm{kg}$ which explains why a geyser regime was always observed. To observe a furamole regime, we had to reduce the water level in the pool, $h$, below a centimeter. 


\subsection{Geyser's death: convection}

In the description of the toy geyser done previously, we have considered that the heat power injected in the system is only transferred to the water inside the reservoir. Actually, thermal convection transfers a part of the injected heat power from the reservoir to the conduit and to the pool. The thermal energy per unit time conveyed in the conduit writes $k\left(T_{r}-T_{b}\right) \pi R^{2}$ with $k$ the heat transfer coefficient in the case of vertical natural convection in a vertical pipe heated from below. This coefficient depends on the Rayleigh number and the Grashof number but stays between 100 and $1000 \mathrm{~W} \cdot \mathrm{K}^{-1} \cdot \mathrm{m}^{-2}$ in the range of parameters encountered in our experiments [39]. The limit case where the convective flux is equal to the injected heat power $\mathcal{P} M$ occurs for a critical conduit radius

$$
R_{c}=\sqrt{\frac{\mathcal{P} M}{\pi k\left(T_{r}-T_{p}\right)}} .
$$

For $\mathcal{P}=220 \mathrm{~W} / \mathrm{kg}, M=1.2 \mathrm{~kg}, k=500 \mathrm{~W} \cdot \mathrm{K}^{-1} \cdot \mathrm{m}^{-2}$ and $T_{r}-T_{p}=70^{\circ} \mathrm{C}$, we get $R_{c}=41 \mathrm{~mm}$. This approach explains why thermal convection in the conduit has been neglected in the model presented in section 3 , where we have $R=3.0 \mathrm{~mm}$. Indeed, in such a situation the convective flux is about 200 times smaller than the injected heat power. Finally, one expects that for a conduit radius larger than $R_{c}$, the injected heat power will be transferred to the entire volume of water and boiling will occur everywhere. This regime of the toy geyser is called the "boiling spring" regime in the rest of this paper.

\subsection{Phase diagram}

The four regimes of a toy geyser, i.e. geyser, fumarole, hot spring and boiling springs are gathered in a single diagram presented in Fig. 7. Such a diagram shows the behavior of a toy geyser depending on the injected heat power $\mathcal{P} M$ and the conduit radius $R$. If the specific heating power is larger than the critical one $\mathcal{P}_{c}$ corresponding to Eq. (10) (White solid line in Fig. 7), the setup exhibits a fumarole regime. Otherwise, the system behaves as a geyser until reaching a critical point, defined by the limit of bubble clogging (Eq. (9) and white dashed line). Below this limit, the setup starts to behave as a hot spring. For even lower heat power $\mathcal{P} M$ or higher conduit radius $R$, the system reaches the limit of heat convection (Eq. 11) and starts to behave as a boiling spring. We indicate in Fig. 7 the range of experimental parameters used for the experiments detailed in Section 2 (white bar) and Section 4.1 (black bar), where a geyser regime and a hot spring regime have been reported.

While the phase diagram in Fig. 7 gives an easy grasp of the different regimes accessible by a geyser-like geometry and the physical parameters governing these regimes, most of natural geysers exhibit more complex dynamics that depend on their own specificities (i.e. change in water inflow, pressure, tectonic stress, etc.. [16, 18]). Another parameter, often discussed, but difficult to characterize in situ, is the interconnection between several geyser reservoirs. Indeed, the interconnection between the plumbing systems of different geysers is known to induce a chaotic and complex dynamics of eruptions [40]. While focusing on the influence of these localized parameters is important for a better characterization of each geyser, pinpointing natural geysers in the present phase diagram would allow to give an idea of how well a toy geyser reproduces the geyser mechanism and what are the geysers specificities that really differentiate natural geysers from their toy models. We hope that future characterizations of geothermal systems, possibly involving geophysics students, will allow such a comparison and will determine to what extent the mechanics of natural pool geysers and toy geysers are similar.

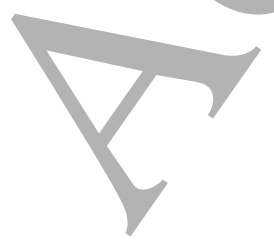




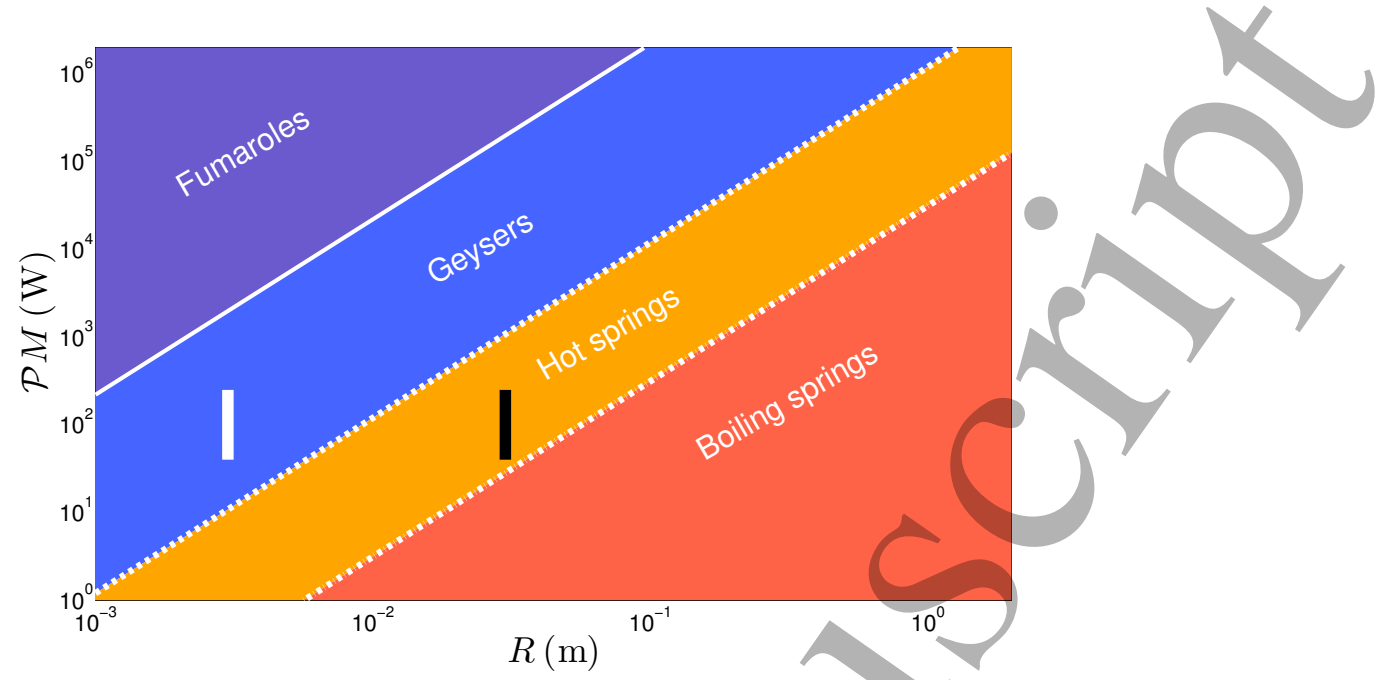

Figure 7. Phase diagram of the behavior of a toy geyser as sketched in Fig. 1(c) as a function of the conduit radius $R$ and the injected heat power $\mathcal{P} M$. The white solid line represents the transition between a fumarole and a geyser regime as predicted by Eq. (10) for $h=4 \mathrm{~cm}$. The dashed line indicates the limit between a geyser and a hot spring regime according to Eq. (9). The dotted line corresponds to the transition between a hot spring and a boiling spring regime as predicted by Eq. (11). The white vertical stripe indicates the range of experimental parameters in Section 2 where a geyser regime has been reported. The dark stripe shows the range of experimental parameters in section 4.1 where a hot spring regime has been observed.

\section{Towards complex geysers: Two connected geysers.}

A way to better link the simplified model that is a toy geyser and a natural geyser is to implement natural observations to the toy geyser experiment. This has been done previously to study the influence of a bubble trap in the vertical conduit of geysers [11]. To approach the complex stochastic eruption of certain natural geysers, we built a setup made of two interconnected reservoirs. Both reservoirs received a different specific heating power $\left(\mathcal{P}_{l}\right.$ and $\left.\mathcal{P}_{r}\right)$ and they were connected to a single pool as sketched in Fig. 8. The connection between the conduits of the two reservoirs is made at a fraction $\alpha$ of the height $H$ of the water column, i.e. $\alpha=0.2$ means that the connection is located at $0.2 \mathrm{H}$ below the pool surface.

\subsection{Experimental data}

Figure 9 shows the time evolution of the temperature in each reservoir of an interconnected toy geyser. The saw-tooth evolution of the temperature in a reservoir is not periodic, contrary to the single reservoir geyser inspected previously. The aim of this section is to describe and rationalize the dynamics of toy geyser with two coupled reservoirs. In the following, we set up our system such that the eruption duration $\tau_{e}$ is small compare to the sleeping time $\tau_{h}$. Under this condition, $\tau \approx \tau_{h}$.

A way to present the data is to plot the time between the eruptions of an event numbered $N$ as a function of the time between the eruptions of the previous event (numbered $N-1$ ). Such a procedure allows to visualize the correlation between successive eruptions and is reported in Fig. 10(a-c) for three different fraction of joined conduit $\alpha$. The figure shows the time lag for each reservoir (red dots and blue squares for left and right reservoirs respectively) and for the total system (gray diamonds). One observes in Fig. 10(a) that even for small fraction of joined conduit $\alpha=0.20$, the time between eruptions in each reservoir undergoes a large dispersion 


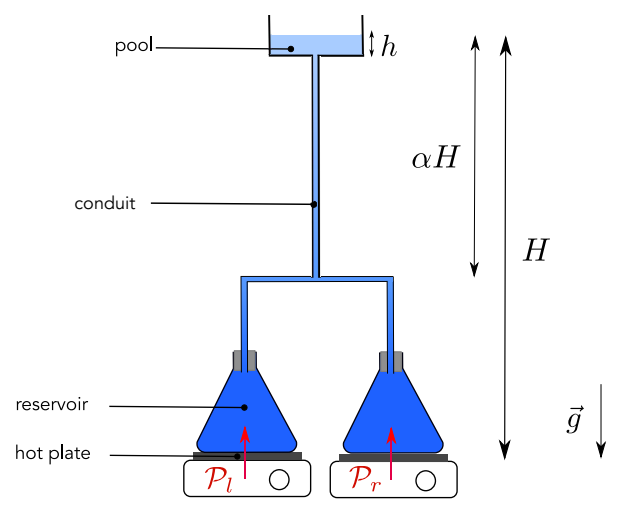

Figure 8. Sketch of a toy geyser experiment with two interconnected reservoirs. The specific heating power received by each reservoir is respectively $\mathcal{P}_{l}$ and $\mathcal{P}_{r}$. The two conduits are connected at a distance $\alpha H$ from the top.

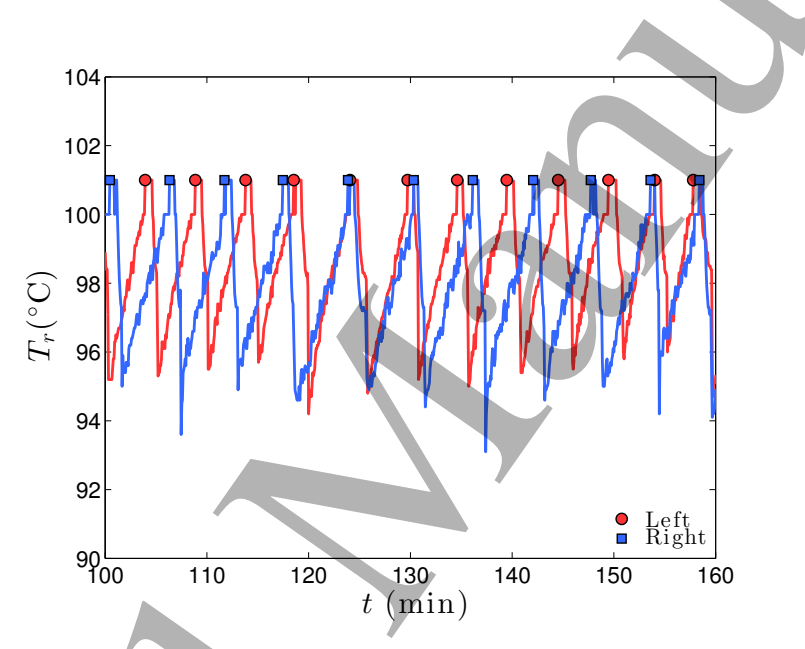

Figure 9. Measured temperatures as a function of time in each reservoir of an interconnected toy geyser as sketched in Fig. 8. The total height of the geyser is $H=1.60 \mathrm{~m}$ and $\alpha=0.20$. The specific heat power injected in the left and right reservoirs are respectively $\mathcal{P}_{l}=110 \mathrm{~W} / \mathrm{kg}$ and $\mathcal{P}_{r}=100 \mathrm{~W} / \mathrm{kg}$. Symbols indicate the time of eruptions.

(about two minutes of dispersion while the mean period is about six minutes). The data for the whole system (gray diamonds) cover a trapezoidal-shaped domain. When $\alpha$ increases to $\alpha=0.70$ [Fig. 10(b)], the dispersion for each reservoir increases and the domain of the global eruption adopt a "banana" shape. Finally, for large fraction of joined conduit $\alpha=0.90$, Fig. 10(c) indicates a reduction of the dispersion of the time between eruptions in each reservoir and for the whole system.

\subsection{Model}

When a reservoir reaches its boiling temperature and erupts, it empties its conduit and reduces the height of the water column of the other reservoir. Thus, if the second reservoir was at a temperature high enough, the reduction of the height of the water column may generate boiling and entrain the eruption of the second reservoir as well. To model this behavior, we assume that the temperature of each reservoir follows a perfect saw-tooth evolution that is governed successively by the equations $d T_{r} / d t=M \mathcal{P} / c$ and $d T_{r} / d t=-M \mathcal{P}_{d} / c$ in order to stay in the 
(a)

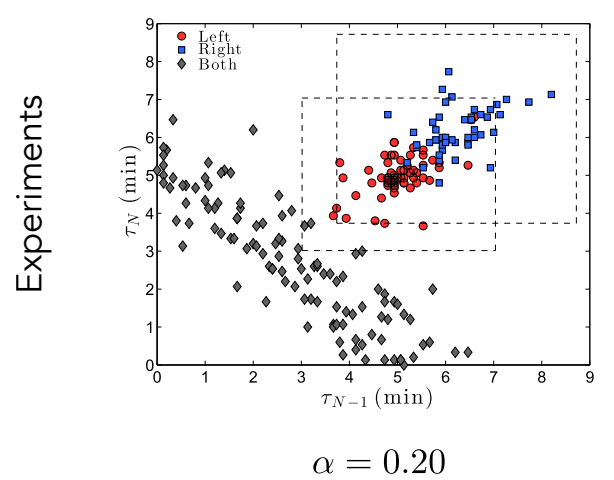

(d)

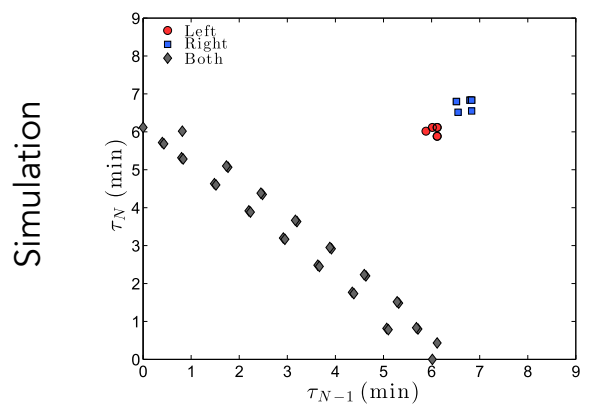

(b)

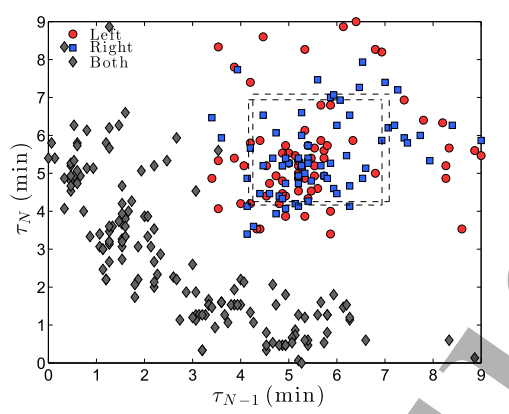

$\alpha=0.70$

(e)

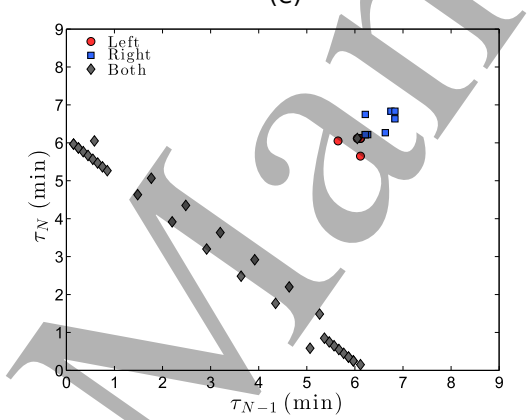

(c)

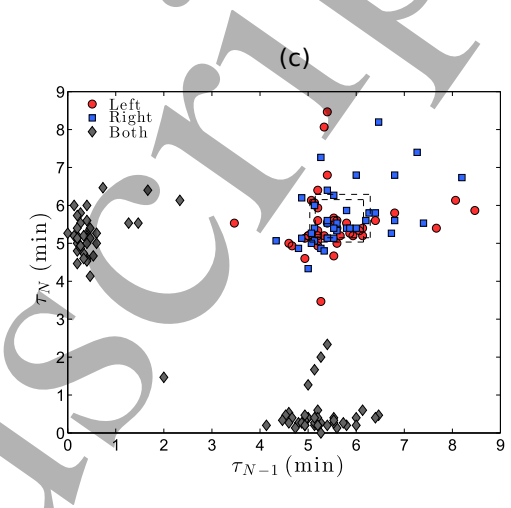

$\alpha=0.90$

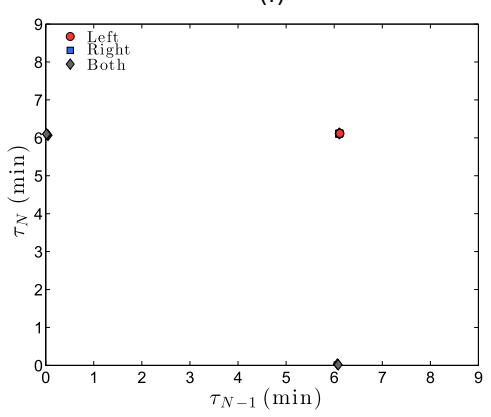

Figure 10. Time between eruptions of the event numbered $N$ and the previous event numbered $N-1$. Red dots and blue squares indicate the time between eruption in each reservoir whereas gray diamonds represent the time between eruptions for the total system. The three top panels correspond to experimental results and the three bottom panels to numerical simulations. The three panels from left to right show different values of $\alpha$, the fraction of joined conduit of the system: (a) (d) $\alpha=0.20$, (b) (e) $\alpha=0.70$ and (c) (f) $\alpha=0.90$. The dashed rectangles show the predictions for the dispersion for the time between eruptions based on the model introduced in Section 5.2.

Figures 10(d-f) show the correlation plot of the times between eruptions for the three values of the coupling parameters inspected experimentally in Section 5.1. It is noticed in Figs. 10(df) that despite the fact that the large spreading of the experimental data is not reproduced by the model, the general trends are similar to the ones observed in section 5.1. Indeed, the times between eruptions of the total system occupy a trapezoidal domain that vanishes at large coupling parameters $\alpha$. Moreover, the spreading of the times between eruptions for each reservoir increases with $\alpha$ up to a critical value where the two reservoirs act as a single one.

The dispersions observed in Figs. 10(a-c) can be approached by the theory developed in Section 3 for a toy geyser with a single reservoir. Equations (3) and (4) yield the time between two eruptions of a reservoir if the other one does not experience any eruption. The substitution into Eqs. (3) and (4) of the boiling temperature $T_{b}(H)$ by its value $T_{b}((1-\alpha) H)$ when the other reservoir has erupted, provides the minimal time between eruptions that a reservoir can follow. The difference between these two extreme cases provides an estimation of the dispersion 
observed for $\tau$. We delimited in Fig. 10(a-c) the domain during which the time between erùptions is supposed to be confined according to this approach. Beyond this first explanation for the data dispersion, other phenomenon can be invoked such as the turbulent gas flow inside the conduit and boiling processes in the reservoir but their theoretical modeling are out of the scope of the present study.

\section{Conclusions}

The study of a toy geyser reveals the successive operations that lead to periodic eruptions of steam and hot water. In a geyser, a geometrical constriction prevents thermal convection of water from a heated reservoir toward a pool. Thus, the water in the reservoir slowly accumulates heat energy by being superheated relatively to the pool level. Such a situation holds until the water in the reservoir boils and the vaporization ejects the water in the conduit, a phenomenon that generates a large release of energy in a short period of time. The slow accumulation of energy and its fast release is characteristic of catastrophic natural events such as earthquakes, volcanic eruptions or thunderbolts. We showed with the example of a toy geyser that there is a strict set of physical parameters that allows an accumulation of thermal energy and a periodic release. Moreover, toy geyser experiments allow us to understand their sensitivity towards external parameters such as water inflow, barometric pressure or pool temperature.

A major difference exists between toy geysers and natural ones: the latter generally adopt a complex dynamics that strongly differs from the clock-like precision of toy geysers. The complexity of real geysers has been approached with a toy geyser having two interconnected reservoirs. Such a setup showed a complex time behavior that has been studied statistically and rationalized theoretically. The rareness of regular geysers in nature reveals that very few of them have a single and independent reservoir. Interconnected and complex plumbing systems seem to prevail in nature leaving room for complexity rather than simplicity.

The present study leaves several open questions such as a deeper understanding of the phenomenon of bubble clogging in confined situations or the exact description of heat transfer during a boiling process in a superheated liquid. The issue of whether a natural geyser with a large conduit radius can operate thanks to a bubble clogging phenomenon remains open. This work opens new avenues for future research about geysers made with a liquid differing from water, or with a liquid inducing solid deposition in order to study the stability of the plumbing system over time.

\section{Acknowledgments}

This research has been funded by the Inter-university Attraction Poles Programme (IAP 7/38 MicroMAST) initiated by the Belgian Science Policy Office. SD thanks the FNRS for financial support. MB acknowledges support as a FNRS-FRIA Fellow. We are grateful to Alice Dubus who realised the glass reservoirs we dreamed about. Tadd Truscott and Zhao Pan are thanked for their fascinations concerning this subject and the excellent idea of interconnecting two reservoirs. The authors acknowledge Hervé Caps, Christophe Clanet, Laurent Maquet, Julien Schokmel, Alexis Duchesne for eruption of ideas and precious comments concerning this work. Finally, the thank anonymous referees for their contributions to improve the quality of this article.

\section{References}

[1] Rinehart J 1969 Bulletin Volcanologique 33 153-163

[2] Azzalini A and Bowman A 1990 Applied Statistics 357-365

[3] Bunsen R 1904 Gesammelte Abhandlungen von Robert Bunsen: im auftrage der Deutschen Bunsengesellschaft für angewandte Physikalische Chemie hrsg vol 1 (W. Engelmann)

[4] Tyndall J 1865 Heat considered as a mode of motion: being a course of twelve lectures delivered at the Royal Institution of Great Britain in the season of 1862 (Longman, Green, Longman, Roberts, \& Green)

5] Saptadji N 1995 Modeling of geysers Ph.D. thesis ResearchSpace, Auckland 
[6] Flieller J B, Suvanto E, Lohner F and Queval M 2017 Journal of Geoscience and Environment Protection 5 194

[7] Lasič S 2006 European journal of physics $\mathbf{2 7} 995$

[8] Steinberg G S, Merzhanov A G and Steinberg A S 1981 Modern Geology 8 67-70

[9] Steinberg G S, Merzhanov A G, Steinberg A S and Rasina A A 1982 Mod. Geol 8 71-74

[10] Toramaru A and Maeda K 2013 Journal of Volcanology and Geothermal Research 257 227-239

[11] Adelstein E, Tran A, Muñoz-Saez C, Shteinberg A and Manga M 2014 Journal of Volcanology and Geothermal Research 285 129-135

[12] Hutchinson R, Westphal J and Kieffer S 1997 Geology 25 875-878

[13] Namiki A, Muñoz-Saez C and Manga M 2014 Journal of Geophysical Research: Solid Earth 119 6229-6248

[14] Munoz-Saez C, Manga M, Hurwitz S, Rudolph M L, Namiki A and Wang C Y 2015 Journal of Volcanology and Geothermal Research 292 41-55

[15] Honda K and Terada T 1906 Physical Review (Series I) 22300

[16] Rinehart J 1972 Journal of Geophysical Research 77 342-350

[17] Hurwitz S, Kumar A, Taylor R and Heasler H 2008 Geology 36 451-454

[18] Hurwitz S, Sohn R A, Luttrell K and Manga M 2014 Journal of Geophysical Research: Solid Earth 119 $1718-1737$

[19] Rojstaczer S, Galloway D, Ingebritsen S and Rubin D 2003 Geophysical research letters 30

[20] Lu X, Watson A, Gorin A V and Deans J 2006 Geothermics 35 409-427

[21] Lu X and Kieffer S W 2009 Annual Review of Earth and Planetary Sciences 37 449-477

[22] Dowden J, Kapadia P, Brown G and Rymer H 1991 Journal of Geophysical Research: Solid Earth (19782012) 96 18059-18071

[23] Rudolph M, Manga M, Hurwitz S, Johnston M, Karlstrom L and Wang C Y 2012 Geophysical Research Letters 39

[24] OHara K and Esawi E 2013 GSA Today 23 4-9

[25] Tyndall J 1881 Heat: a mode of motion (D. Appleton)

[26] Lide D 2004 CRC handbook of chemistry and physics (CRC press)

[27] Flieller J, Suvanto E, Lohner F and Queval M 2017 Journal of Geoscience and Environment Protection 5 $194-226$

[28] Pinhasi G A, Ullmann A and Dayan A 2005 Reviews in Chemical Engineering 21 133-264

[29] Kaichiro M and Ishii M 1984 International Journal of Heat and Mass Transfer 27 723-737

[30] Haberman W and Morton R 1953 An experimental investigation of the drag and shape of air bubbles rising in various liquids Tech. rep. DTIC Document

[31] Mishima K and Hibiki T 1996 International Journal of Multiphase Flow 22 703-712

[32] Vergniolle S and Mangan M 2000 Encyclopedia of volcanoes 447-461

[33] Han W, Lu M, McPherson B, Keating E, Moore J, Park E, Watson Z and Jung N H 2013 Geofluids 13 283-297

[34] Coffey T 2008 American Journal of Physics 76 551-557

[35] Rodríguez-Rodríguez J, Casado-Chacón A and Fuster D 2014 Physical review letters 113214501

[36] Gonnermann H M and Manga M 2007 Annu. Rev. Fluid Mech. 39 321-356

[37] Walker G P L 1973 Geologische Rundschau 62 431-446

[38] Wilson L 1980 Journal of Volcanology and Geothermal Research 8 297-313

[39] Jackson J, Cotton M and Axcell B 1989 International journal of heat and fluid flow 10 2-15

[40] Nicholl M, Wheatcraft S, Tyler S and Berkowitz B 1994 Journal of Geophysical Research: Solid Earth (1978-2012) 99 4495-4503 